\title{
Quantifying Deformation at the Micro- and Nanoscale Using In Situ Electron and 3D Confocal Microscopy
}

\author{
D.S. Gianola, L. Chen, R.P. McCaffrey, and K. Murphy
}

Department of Materials Science and Engineering, University of Pennsylvania, 3231 Walnut St., Philadelphia, PA 19104

The recent interest in size-dependent deformation of micro- and nanoscale materials has paralleled both technological miniaturization and advancements in imaging and small-scale mechanical testing methods [1,2]. Here we describe two distinct quantitative in situ approaches for mico- and nanomechanical testing that make use of both electron and visible light imaging platforms.

The first system, designed and implemented for use in scanning electron and focused ion beam microscopes, employs either a transducer based on a three-plate capacitor system or a microelectromechanical systems platform, which are used for high-fidelity force and displacement measurements [4]. Specimen manipulation, transfer, and alignment are performed using a manipulator, independently controlled positioners, and the focused ion beam. Gripping of specimens is achieved using electron-beam assisted Pt-organic deposition. Local strain measurements are obtained using digital image correlation of electron images taken during testing. This system has been applied to the testing of metallic nanowires (Fig. 1), nanoporous micropillars, and shape memory alloy microspecimens.

A distinct small-scale specimen testing apparatus installed within a high speed confocal microscopy system (Fig. 2) will also be discussed. This system is capable of providing full 3D reconstruction, visualization, and deformation analysis. The testing system employs piezoelectric actuators, highfidelity load cells, and multi-axis alignment stages. Digital volume correlation, which relies on computation of cross-correlation coefficients of sub-volumes of digital images, is utilized to provide local and full-field displacement and strain measurements in 3D with subvoxel resolution. We will show an application of this novel system to the study of the mechanics of 3D periodic microframed materials to correlate unit-cell level deformation with macroscopic properties.

The utility of these systems will be discussed in the context of the unique mechanical behavior that manifests in small-scale materials. In addition, results highlighting information that can be obtained via cross-platform testing will be shown.

\section{References}

[1] D.S. Gianola, C. Eberl, JOM 61 (2009) 24.

[2] M. Legros, D.S. Gianola, C. Motz, MRS Bull. 35 (2010) 354.

[3] G. Richter et al., Nano Lett. 9 (2009) 3048. 

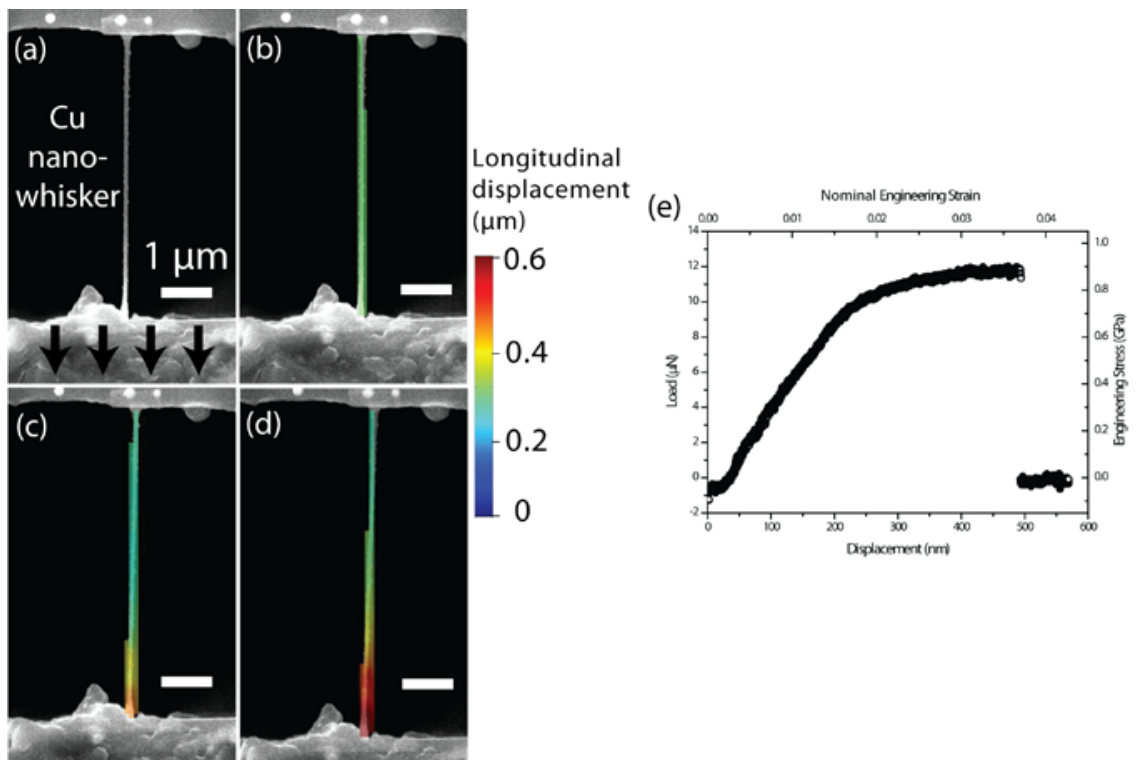

FIG. 1. In situ SEM tensile testing of $\mathrm{Cu}$ nanowhisker with a diameter of $75 \mathrm{~nm}$. (a) $\mathrm{Cu}$ Nanowhisker prior to testing. (b-d) Sequence during tensile testing with superimposed longitudinal displacement fields computed using digital image correlation. (e) Tensile load-displacement curve for an individual single crystalline Au nanowhisker with a diameter of $133 \mathrm{~nm}$.
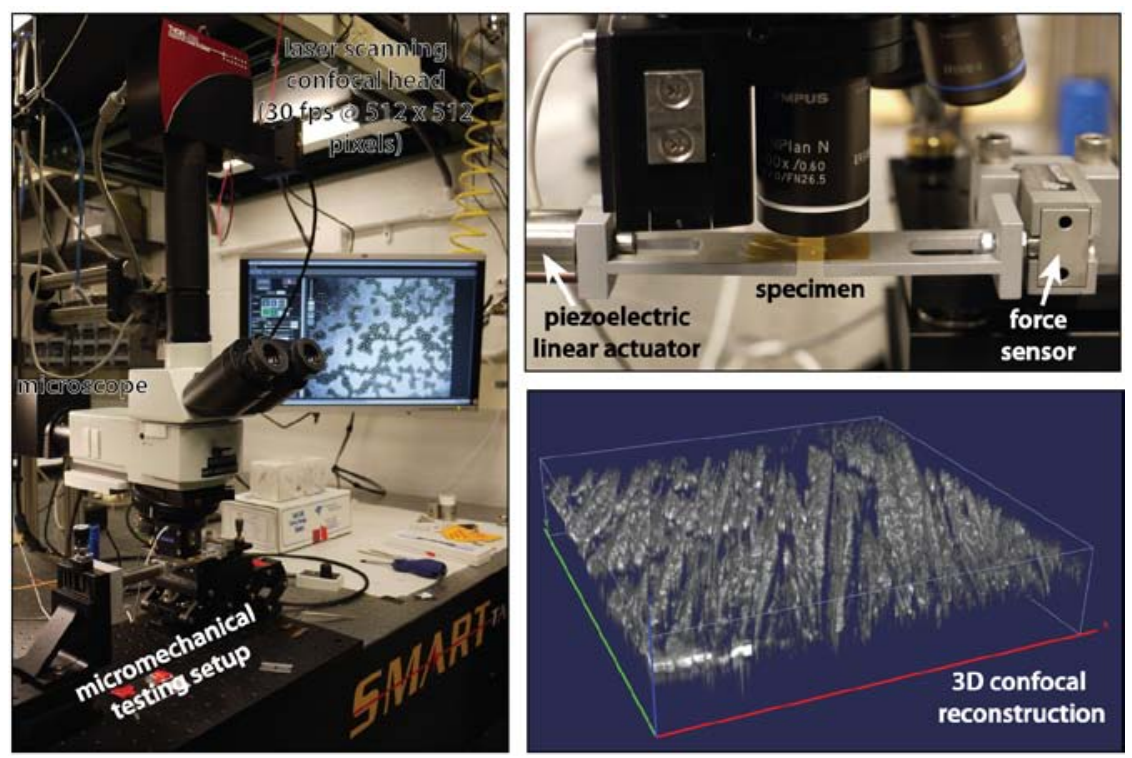

FIG. 2. 3D micromechanical testing setup, which utilizes (i) custom-built mechanical testing platform, (ii) laser scanning 3D confocal microscope, and (iii) digital volume correlation software for 3D strain and displacement measurements. 